\title{
Why are parents in Canada less likely to breastfeed than those in poorer nations?
}

\author{
Cite as: CMAJ 2020 February 24;192:E197-8. doi: 10.1503/cmaj.1095849
}

Posted on cmajnews.com on February 7, 2020

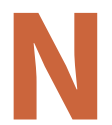

o countries fully meet internationally accepted recommendations for breastfeeding of infants. But wealthy nations like Canada are further from achieving these goals than many poorer countries.

More than one in five infants are never breastfed in wealthier countries, compared to just one in 25 infants in poorer countries, according to UNICEF.

Paradoxically, within Canada, the poorest parents are the least likely to breastfeed. This means that Canadian mothers who have the greatest difficulty in affording infant formula are the ones most likely to use it.

The World Health Organization (WHO) recommends that parents exclusively feed their children breastmilk for the first six months of life, starting within one hour of birth, and continue giving them breastmilk until at least two years after birth.

The benefits to babies are well known, with breastfed infants being less vulnerable to infection and malnutrition, as well as some chronic diseases, like type 2 diabetes, later in life. Parents who breastfeed also benefit, with faster recovery and weight loss after giving birth, lower risks of breast and uterine cancers, and reduced likelihood of early menopause, as shown by new research.

In countries like Madagascar, Nepal, and Sri Lanka, more than $99 \%$ of infants are breastfed, compared to 89\% in Canada. Partly this difference comes down to access to formula - in poorer countries, many parents cannot afford a consistent supply. But these countries also have cultures and policies that support breastfeeding.

According to WHO reports, virtually all infants in Sri Lanka are born in hospitals

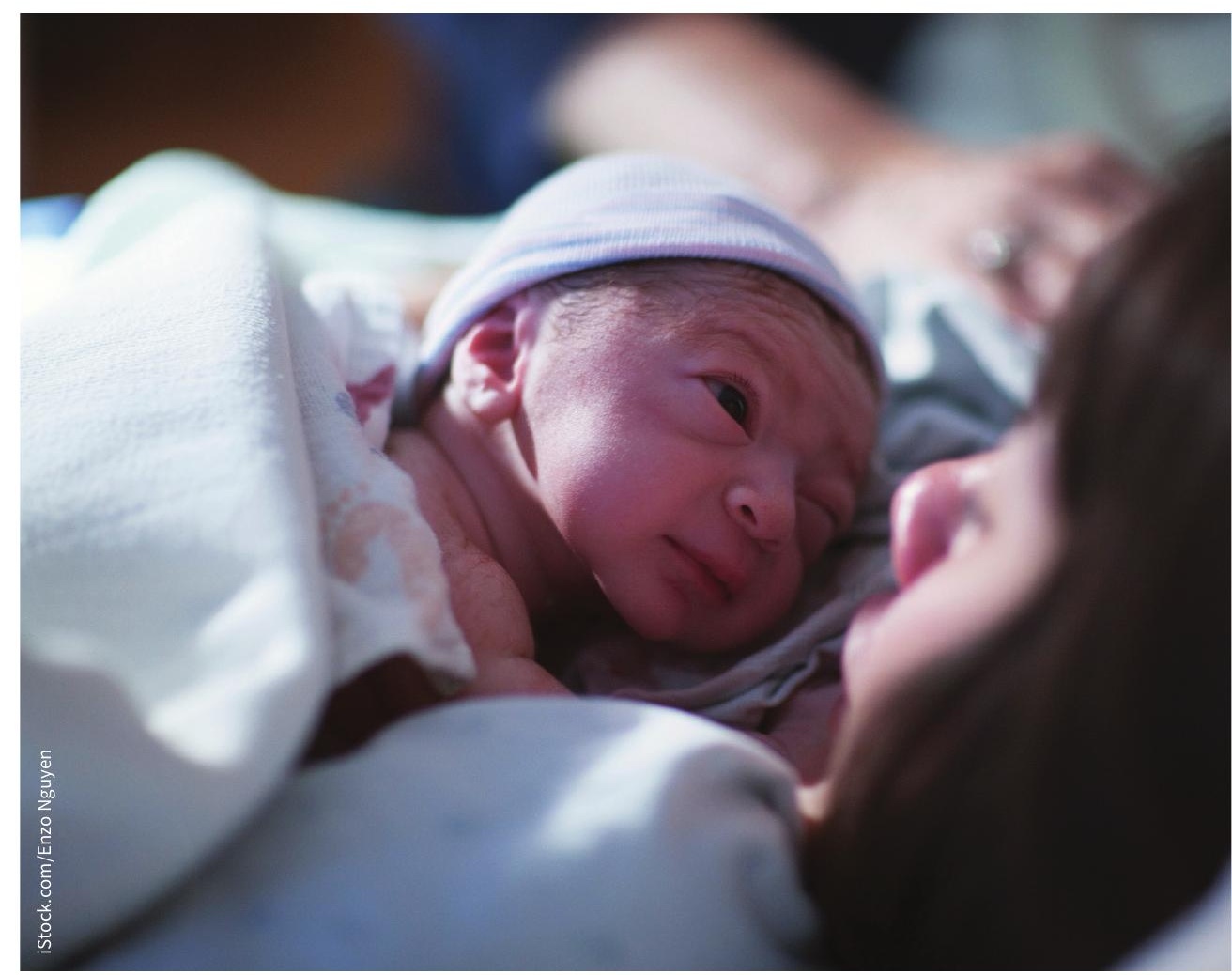

Many low- and middle-income countries have higher breastfeeding rates than Canada.

and clinics that meet Baby-Friendly Hospital Initiative standards aimed at promoting breastfeeding and close relationships between parents and babies. And in Madagascar, $88 \%$ of hospitals and clinics are designated Baby-Friendly.

In contrast, just $2 \%$ of hospitals and clinics in Canada have a Baby-Friendly designation, despite support from groups like the Canadian Paediatric Society.

Owen Sound Hospital is one of the few Canadian facilities with this designation; it now has a $92 \%$ breastfeeding rate after delivery, up from $73 \%$ in 2013, the last year before it became a Baby-Friendly hospital.
One of the prerequisites for a BabyFriendly designation is enforcing the WHO code on limiting marketing of breastmilk substitutes, including free samples and inhospital promotion of formula. Madagascar, Nepal and Sri Lanka have all fully incorporated the code into law.

But Canada has not cracked down on the marketing of formula in almost four decades since the introduction of the WHO code. Companies that make formula face few restrictions, self-imposed or legal. One company, Nestlé Canada, says it "voluntarily and unilaterally applies the WHO Code in all developing countries," 
although aid agencies have accused its parent company of violating the code.

"It's hard not to see the effects of marketing in terms of how it has gradually eaten away at the whole value of breastfeeding," says Dr. Nigel Rollins of the WHO Department of Maternal, Newborn, Child and Adolescent Health. "Many women will say they appreciate the information that they get from these marketing sites on infant feeding because they are not getting information and support from anywhere else."

In Canada, some social supports for Indigenous families may unintentionally promote bottle-feeding by routinely providing formula, says Jaime Cidro, an anthropology professor at the University of Winnipeg. Indigenous parents may also experience discrimination when they give birth in hospitals, often far away from their home communities.

"They wouldn't necessarily have all that family support in those first few days when you really need to get a handle on breastfeeding," Cidro says. "In many cases, women are travelling home shortly after they give birth, in buses, travelling for long distances in ways that also impede breastfeeding."

Canada has also seen a backlash against "breast is best" messaging in recent years, as more parents have spoken out about the social pressure they faced because they were unable to breastfeed or chose to bottle-feed.
A supportive environment is essential to reach higher breastfeeding rates. But all new parents need support, not just those who breastfeed, says Dr. Jessica Ranjani, a Toronto-based pediatrician and mother of two. "Caring for a new baby is an extremely difficult job," she says. "The culture needs to be supportive of that and acknowledge that, as opposed to making more expectations."

According to Owen Sound registered nurse Colleen Ford, "we would still give the same level of education and care for a mother who has come in and decided to formula feed as a mother who is breastfeeding."

Allison Daniel, Toronto, Ont. 